CERN/EP 98-80

April 20, 1998

\title{
Development of CVD Diamond Radiation Detectors *
}

\section{The RD42 Collaboration}

W. Adam ${ }^{1}$, C. Bauer ${ }^{2}$, E. Berdermann ${ }^{3}$, F. Bogani ${ }^{4}$, E. Borchi ${ }^{5}$, M. Bruzzi ${ }^{5}$, C. Colledani ${ }^{6}$, J. Conway ${ }^{7}$, W. Dabrowski ${ }^{8}$, P. Delpierre ${ }^{9}$, A. Deneuville ${ }^{10}$, W. Dulinski ${ }^{6}$, B. van Eijk ${ }^{11}$, A. Fallou ${ }^{9}$,

D. Fish ${ }^{7}$, F. Foulon ${ }^{12}$, M. Friedl ${ }^{1}$, K.K. Gan ${ }^{13}$, E. Gheeraert ${ }^{10}$, E. Grigoriev ${ }^{2}$, G. Hallewell ${ }^{9}$, R. Hall-Wilton ${ }^{14}$, S. Han ${ }^{15}$, F. Hartjes ${ }^{11}$, J. Hrubec ${ }^{1}$, D. Husson ${ }^{6}$, H. Kagan ${ }^{13}$, D. Kania ${ }^{16}$, J. Kaplon ${ }^{8}$, R. Kass ${ }^{13}$, K.T. Knöpfle ${ }^{2}$, M. Krammer ${ }^{1}$, P.F. Manfredi ${ }^{17}$, D. Meier ${ }^{8, \dagger}$, M. Mishina ${ }^{18}$, F. LeNormand ${ }^{6}$, L.S. Pan $^{19}$, H. Pernegger ${ }^{1}$, M. Pernicka ${ }^{1}$, S. Pirollo ${ }^{5}$, V. Re ${ }^{17}$, J.L. Riester ${ }^{6}$, S. Roe ${ }^{8}$, D. Roff ${ }^{14}$,

A. Rudge ${ }^{8}$, S. Schnetzer ${ }^{7}$, S. Sciortino ${ }^{5}$, V. Speziali ${ }^{17}$, H. Stelzer ${ }^{3}$, R. Stone ${ }^{7}$, R.J. Tapper ${ }^{14}$,

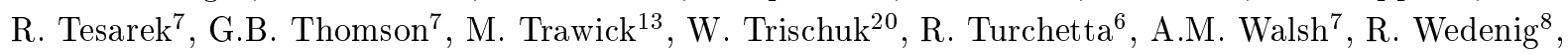
P. Weilhammer ${ }^{8}$, H. Ziock ${ }^{15}$, M. Zoeller ${ }^{13}$

${ }^{1}$ Institut für Hochenergiephysik der Österr. Akademie d. Wissenschaften, A-1050 Vienna, Austria

${ }^{2}$ MPI für Kernphysik, D-69029 Heidelberg, Germany

${ }^{3}$ GSI, Darmstadt, Germany

${ }^{4}$ LENS, Florence, Italy

${ }^{5}$ University of Florence, Florence, Italy

${ }^{6}$ LEPSI, CRN, Strasbourg 67037, France

7 Rutgers University, Piscataway, NJ 08855, U.S.A.

${ }^{8}$ CERN, CH-1211, Geneva 23, Switzerland

${ }^{9}$ CPPM, Marseille 13288, France

10 LEPES, Grenoble, France

11 NIKHEF, Amsterdam, Netherlands

12 Centre d'Etudes de Saclay, 91191 Gif-Sur-Yvette, France

13 The Ohio State University, Columbus, OH 43210, U.S.A.

${ }^{14}$ Bristol University, Bristol BS8 1TL, U.K.

${ }^{15}$ Los Alamos National Laboratory, Research Division, Los Alamos, NM 87545, U.S.A.

${ }^{16}$ Lawrence Livermore National Laboratory, Livermore, CA 94550, U.S.A.

17 Universita di Pavia, Dipartimento di Elettronica, 27100 Pavia, Italy

18 FNAL, Batavia, U.S.A.

19 Sandia National Laboratory, Livermore, CA 94550, U.S.A.

${ }^{20}$ University of Toronto, Toronto, ON MSS 1A7, Canada

\begin{abstract}
Diamond is a nearly ideal material for detecting ionizing radiation. Its outstanding radiation hardness, fast charge collection and low leakage current allow a diamond detector to be used in high radiation, high temperature and in aggressive chemical media. We have constructed charged particle detectors using high quality CVD diamond. Characterization of the diamond samples and various detectors are presented in terms of collection distance, $d=\mu E \tau$, the average distance electron-hole pairs move apart under the influence of an electric field, where $\mu$ is the sum of carrier mobilities, $E$ is the applied electric field, and $\tau$ is the mobility weighted carrier lifetime. Over the last two years the collection distance increased from $\sim 75 \mu \mathrm{m}$ to over $200 \mu \mathrm{m}$. With this high quality CVD diamond a series of micro-strip and pixel particle detectors have been constructed. These devices were tested to determine their position resolution and signal to noise performance. Diamond detectors were exposed to large fluences of pions, protons and neutrons to establish their radiation hardness properties. The results of these tests and their correlation with the characterization studies are presented.
\end{abstract}

\footnotetext{
${ }^{*}$ presented at $5^{\text {th }}$ Int. Symposium on Diamond Materials, Paris, (1997).

${ }^{\dagger}$ corresponding author D. Meier: e-mail Dirk.Meier@cern.ch
} 


\section{Introduction}

Solid state tracking devices have become one of the main stays of general purpose high energy physics detectors. Detectors in future high energy and nuclear collider experiments will be exposed to high radiation levels. At a distance of $10 \mathrm{~cm}$ from the beam axis, for instance, detectors are expected to receive a fluence of $1.6 \times 10^{15}$ particles per $\mathrm{cm}^{2}$ during 10 years of operation at the Large Hadron Collider (LHC) at the European Laboratory of Particle Physics (CERN). There are very few materials which can withstand this level of radiation. CVD diamond is a radiation resistant detector material which may be able to operate close to the interaction region at future experiments.

The RD42 collaboration is working as a detector R\&D project on the development of diamond detectors for particle tracking at LHC. We study CVD diamond with regard to their electrical material properties, their radiation hardness and tracking performance and participate in the development of fast and radiation hard readout electronics. We have built various diamond sensors and tracking devices in strip or pixel geometries which have been studied with readout electronics presently in use or under development for LHC.

The CVD diamond substrates are manufactured in industry in size and geometry comparable to silicon detectors. Different from silicon detectors, they operate in high radiation environment at room temperature with negligible leakage current and negligible power dissipation. Noise due to leakage current is negligible in the insulating diamond detector. The capacitance of diamond is low due to relatively low electrical permeability resulting in a reduced noise. The low proton number of diamond causes relatively small multiple scattering of particles which is important for particle tracking and exact identifiction of decay vertices. By working with CVD diamond manufacturers we have been able to improve the diamond substrate over the last few years.

In the following we are reporting the work on diamond characterization, performance of a characteristic tracking device and studies on neutron, pion and proton irradiations.

\section{Charge Collection Properties of Diamond Detectors}

We characterize CVD diamond samples in terms of their charge collection distance which is the distance electrons and holes move in average apart in an external electric field inside the diamond. It is one of the most important characteristics for diamond-based particle detectors because of its relation to fundamental material properties. The distances $d_{e}, d_{h}$ which electrons or holes travel in an electric field $E$ depend on their lifetime $\tau_{e}, \tau_{h}$ and mobility $\mu_{e}, \mu_{h}$.

$$
d_{e}=\mu_{e} \tau_{e} E \quad \text { and } \quad d_{h}=\mu_{h} \tau_{h} E,
$$

The charge collection distance $d$ is the sum of these distances and can be expressed using the summed mobility $\mu=\mu_{e}+\mu_{h}$ and the mobility weighted lifetime $\tau$ of electrons and holes

$$
d=d_{e}+d_{h} \equiv \mu \tau E .
$$

Above a certain field strength the carrier velocities saturate and $d$ is expected to stay constant. It was shown previously, that the charge collection distance varies as a function of depth within a sample and particularly increases from the nucleation to the growth side $[1,2]$. We have expanded this observation by measuring the charge collection distance for high quality diamond after successive material removal from the nucleation side and observe a linear increase in the collection distance. In another study we exposed diamond samples to relatively small doses of radiation and observe an increasing charge collection distance. This "pumping" effect has been observed previously [3]. In the pumped state traps are filled and release their charge during 
either heating or exposure to 'cold' fluorescent light. This release of charge is also observed by luminescence light and thermally stimulated current measurements.

\subsection{Status of Charge Collection Distance}

Fig. 1 shows a basic diamond readout and how its charge signal is measured. The signal can be read on both sides of the detector. The charge signal is either generated by electrons from a source or by high energetic particles from an accelerator. Charged particles traversing the

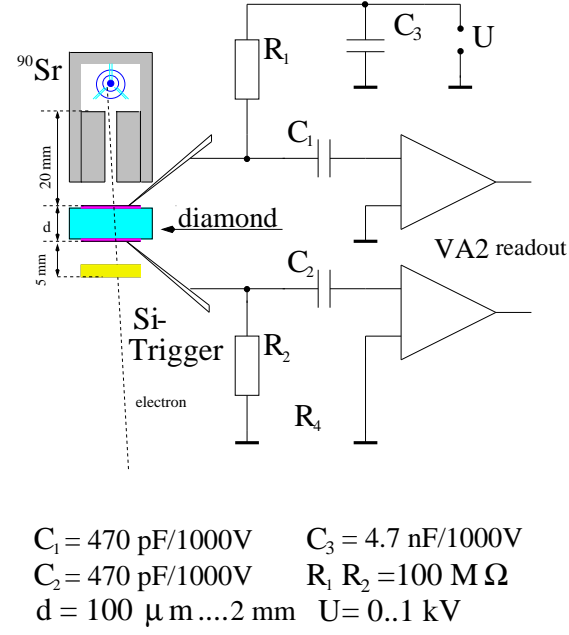

Figure 1: Principle and setup for charge collection measurement in a diamond detector. In the lab charged particles from radioactive sources are used to measure charge collection of the sample The diamond and readout electronics in this configuration is also used for particle tracking in high energetic beams.

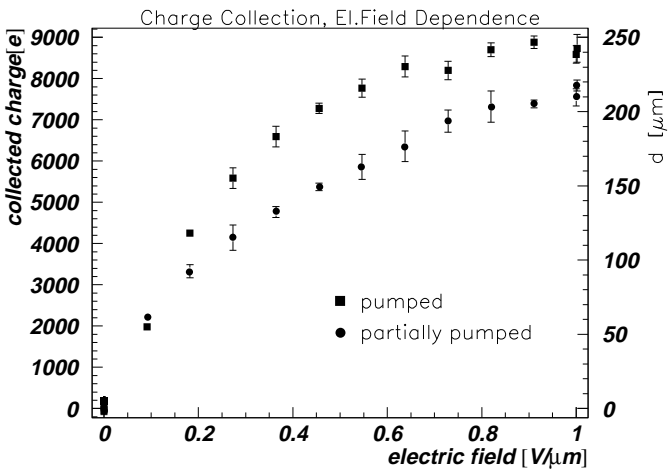

Figure 2: Mean collected charge and charge collection distance on CVD diamond from recent growth as a function of the applied electric field. The charge collection distance on this sample reaches $d=250 \mu \mathrm{m}$.

diamond detector deposit energy and generate electron hole pairs along their path. The charge $Q_{\text {col }}$ which can be collected at the electrodes after one charged particle traversed the diamond characterizes the performance of the detector.

A minimum ionizing particle mip, e.g. an electron with a kinetic energy of $\approx 3 \mathrm{MeV}$, deposits $245 \mathrm{keV}$ in $D=500 \mu \mathrm{m}$ thick diamond. The energy which is necessary to excite one $e h$-pair in diamond is $13.6 \mathrm{eV}$. One obtains the number $\left\langle Q_{m i p, \text { gen }}\right\rangle / D=36 e / \mu \mathrm{m}$ of generated electrons or holes [4]. Electron hole pairs separate in the applied electric field and travel towards the electrodes where they induce a charge $Q_{\text {col }}$. The mean measured charge $\left\langle Q_{\text {col }}\right\rangle$ is related to the charge collection distance $d$ of the diamond bulk and in an approximation one finds [4]

$$
d \approx \frac{\left\langle Q_{\mathrm{col}}\right\rangle}{\left\langle Q_{\text {mip,gen }}\right\rangle / D}=\frac{\left\langle Q_{\mathrm{col}}\right\rangle}{36 e / \mu \mathrm{m}}
$$

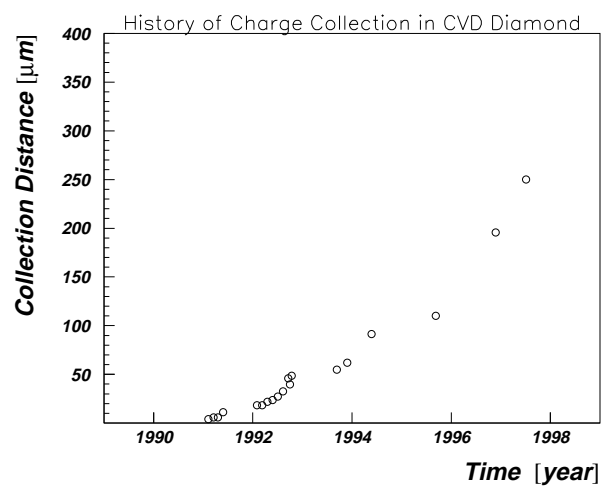

Figure 3: Historical evolution of charge collection in CVD diamond measured with the described method [Fig. 1]. 
which relates the charge collection distance to the collected charge at the electrodes. Fig.2 shows a measurement of the charge collection distance as a function of the applied electric field. The charge collection distance on this samples reaches $d=250 \mu \mathrm{m}$ at $1 \mathrm{~V} / \mu \mathrm{m}$. The samples has a thickness of $1098 \mu \mathrm{m}$. The charge collection saturates between 0.8 to $1 \mathrm{~V} / \mu \mathrm{m}$. Using an electron mobility of $180 \mu \mathrm{m}^{2} / \mathrm{V} / \mathrm{ns}$ and a hole mobility of $120 \mu \mathrm{m}^{2} / \mathrm{V} / \mathrm{ns}$ we estimate for this sample a saturation of carrier velocities at $250 \mu \mathrm{m} / \mathrm{ns}$ and a carrier lifetime of about $1 \mathrm{~ns}$.

Fig. 3 shows the increase of charge collection distance on CVD diamond over the past 7 years and the charge collection of $250 \mu \mathrm{m}$ on available CVD diamond today (1997).

\subsection{Material Removal Study}

CVD diamond typically grows in a polycrystalline columnar structure along the growth direction. The substrate side begins with small grains $(\approx 1 \mu \mathrm{m})$ which grow with material thickness. As the material grows it develops the texture of the fastest growing crystal orientation. It has previously been shown $[1,2]$ that the electrical properties of CVD diamond vary with the thickness of the material: the carrier lifetimes are small on the substrate side and large on the growth side. As a result, the raw diamond material can be "improved" by removing material from the substrate side. This procedure has been used to increase the signal size by $40 \%$ over the as-grown sample. Fig. 4 shows the improvement of the quality of diamond measured by the increase in signal size as a function of the fraction of material removed. The measurement can

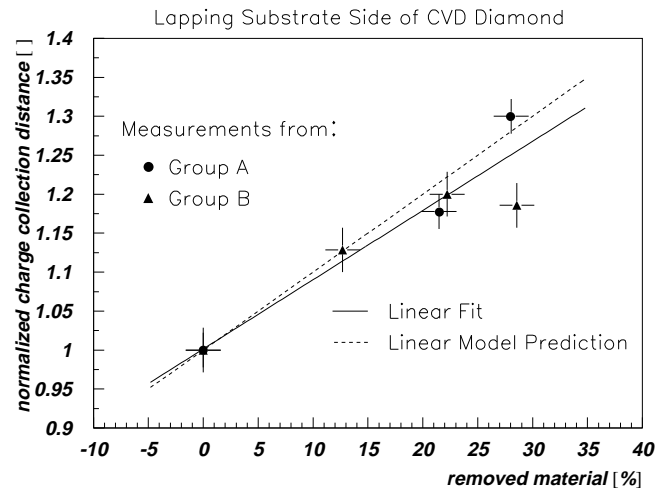

Figure 4: Measured charge collection distance as a function of the material removed from the substrate side. The sample was lapped three times by about $60 \mu \mathrm{m}$ at each step. The charge collection increases after each step which is explained by the linear increase of charge collection from the substrate side in the diamond.

be explained by assuming a linearly increasing charge collection distance $d(x)=c \cdot x$ along the direction of growth $x$ with a slope $c=2 d_{\text {original }} / D_{\text {original }}$ and $d(0)=0$ on the substrate side of the sample. The charge collection distance $\bar{d}_{1}$ after material removal $D_{1}$ from the substrate side leaving a sample of thickness $D=D_{\text {original }}-D_{1}$ is then given by

$$
\bar{d}_{1}=\frac{\int_{D_{1}}^{D_{\text {original }}} d(x) \mathrm{d} x}{\int_{D_{1}}^{D_{\text {original }}} \mathrm{d} x}=d_{\text {original }}\left(1+\frac{D_{1}}{D_{\text {original }}}\right) \quad ; d \ll D_{\text {original }}
$$

The increase of charge collection distance predicted by Eq. 4 is shown in Fig. 4 in good agreement with the measurement.

\subsection{Pumping with Charged Particles}

It is known that the charge collection distance at constant voltage increases under illumination with $\gamma$ or $\beta$ radiation [3,5]. On unirradiated samples an increase by a factor of 1.6 to 2.0 below $\approx 10$ Gy absorbed dose is observed. Above 10 Gy charge collection distance stays constant [Fig. 5]. 
The increase at relatively low dose is called pumping. It is attributed to trap filling which then allows charge carriers to travel longer distances before being traped which is equivalent to an increase in lifetime $\tau$ [Eq. 2]. A sample can be depumped by exposure to fluorescent light and charge collection distance returns to its original value [6]. Depumping is explained by releasing filled traps. The effects of pumping and depumping are reproducible. After exposure to ${ }^{90} \mathrm{Sr}$ of different dose we observe luminescence light and excess current during heating [Fig. 6,7]. The intensity of the luminescence light increases linearly with dose and saturates. Exposure to light decreases luminescence and thermally stimulated current as well as charge collection distance.

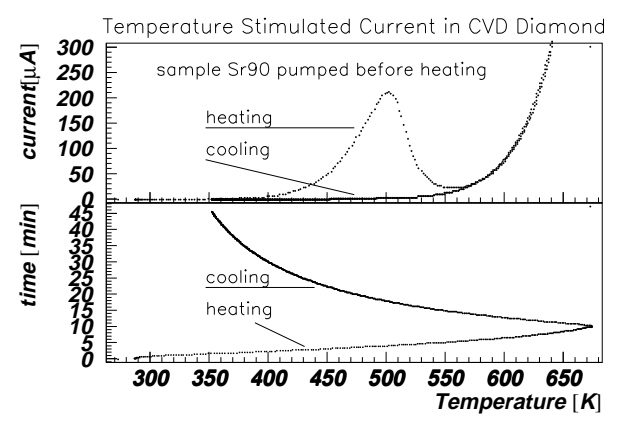

Figure 6: Temperature stimulated current as a function of temperature after exposure to $\beta$ radiation from a ${ }^{90} \mathrm{Sr}$ source. The current increases from a few pico-Ampere at room temperature and $100 \mathrm{~V}$ to several $100 \mu \mathrm{A}$ during heating. Once heated, charge carriers are flushed and the current peak is missing.

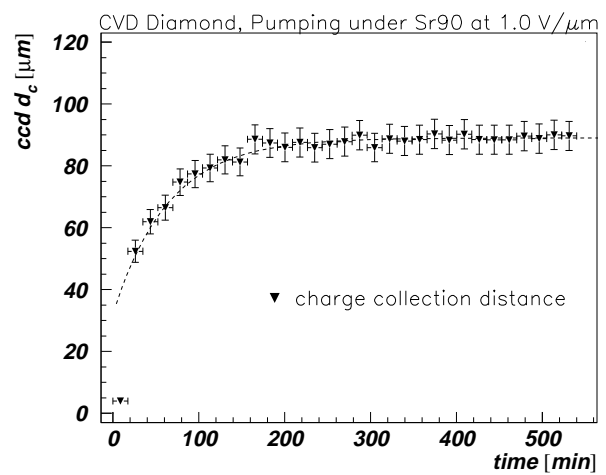

Figure 5: Pumping on a diamond with $\beta$ radiation from a ${ }^{90} \mathrm{Sr}$ source. The charge collection distance increases and saturates under illumination with charged particles.

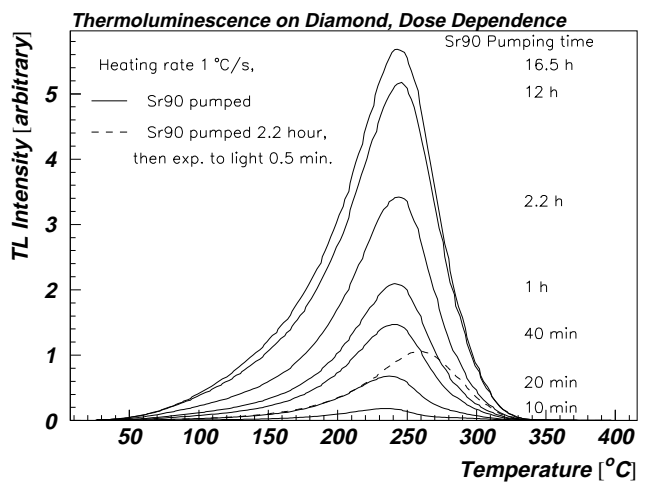

Figure 7: Thermo-luminescence signal as a function of temperature at different doses of $\beta$ radiation from a ${ }^{90} \mathrm{Sr}$ source. The dotted graph shows the thermo-luminescence after one hour pumping with ${ }^{90} \mathrm{Sr}$ followed by an exposure to a fluorescent light lamp.

\section{Tracker Studies}

The first diamond particle tracker was tested in 1993 at CERN [7]. It had $100 \mu \mathrm{m}$ readout pitch and a charge collection distance of $50 \mu \mathrm{m}$. In these tests a $100 \mathrm{GeV} / c$ pion beam $\left(\pi^{-}\right)$ passes through a telescope consisting of eight planes silicon micro-strip detectors. The silicon telescope predicts the pion track within a few micro-meter resolution. The diamond detectors under study are in between the silicon planes. Fig. 8 shows a metalization pattern on a recent strip tracker. The strips have a pitch of $50 \mu \mathrm{m}$. They form ohmic contacts to the diamond. Other patterns like pixel or pad structures were prepared and tested as well. The charge signal on strips is read out using VA2 VLSI chips [8]. The VA2 contains 128 channels of low noise charge integrating preamplifiers followed by a signal shaper. 
The shaped signal has a peaking time of about $2 \mu$ s and in praxis an equivalent noise charge of $80 e+11 e / \mathrm{pF}$. Other readout configurations which suit requirements at the LHC like shorter peaking time and radiation hardness are being studied as well [9]. The charge collection distance on the diamond tracker described here was measured with a ${ }^{90} \mathrm{Sr}$ source in the lab [Fig. 9] and reaches about $210 \mu \mathrm{m}$ at an electric field of $1 \mathrm{~V} / \mu \mathrm{m}$ which corresponds to $7600 e$ collected charge. Fig. 10 shows the distribution of collected charge on this diamond as a strip detector in a $100 \mathrm{GeV} / c$ pion beam. The charge signal is nearly Landau distributed and has a mean value of $8200 e$ depending on the number of strips adjacent to the predicted intersection. The mean collected charge of $8200 \mathrm{e}$ corresponds to a charge collection distance of $230 \mu \mathrm{m}$ which agrees within the measurement error with the ${ }^{90} \mathrm{Sr}$ result. The noise on a strip ideally depends only on the leakage current and strip capacitance. In diamond the contribution due to leakage current is negligible. The capacitance is of a few pico-Farad depending on the strip geometry. We measured a noise of about $150 e$ which gives a mean signal-to-noise ratio for this tracker of 55-to-1. The distribution of the difference between the track prediction perpendicular to the strips and the actual measured position by the diamond detector is nearly gaussian and gives a resolution of $\sigma=$ $16.5 \mu \mathrm{m}$ [Fig. 11] close to the digital resolution expected for a strip detector with $50 \mu \mathrm{m}$ readout pitch $\left(\sigma_{\text {digital }}=50 \mu \mathrm{m} / \sqrt{12} \approx 14.3 \mu \mathrm{m}\right)$.
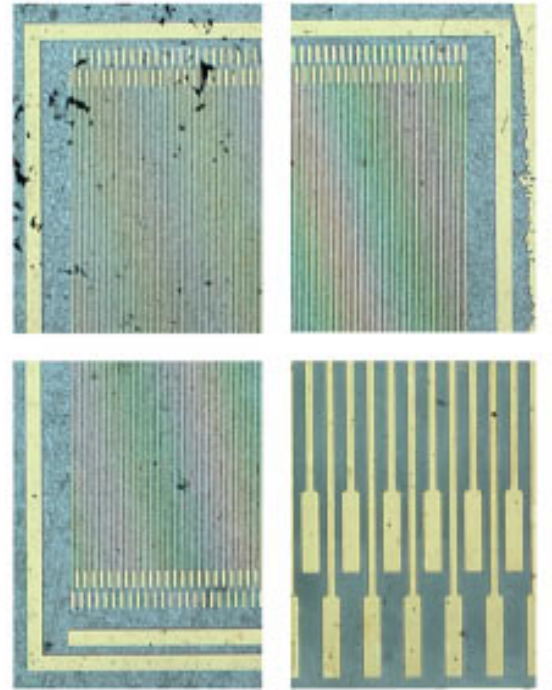

Figure 8: Photo of a diamond strip detector. The strips have a width of $25 \mu \mathrm{m}$ and pitch of $50 \mu \mathrm{m}$. The magnification right at the bottom shows bond pads from were bond wires connect to the readout chip.

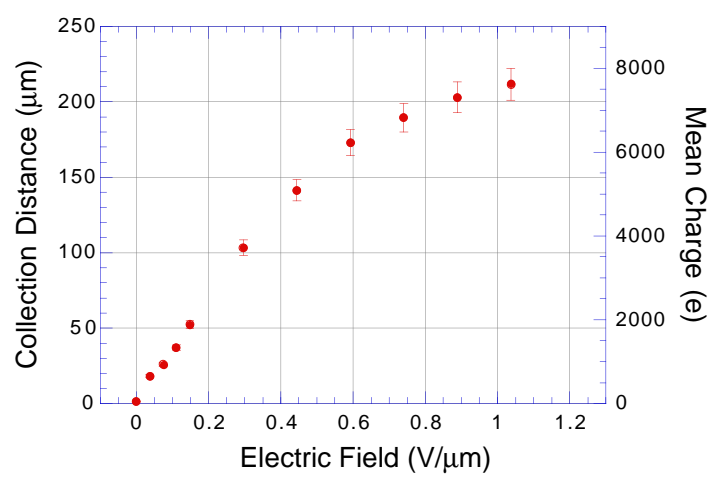

Figure 9: Charge collection distance measured with a ${ }^{90} \mathrm{Sr}$ source and dot electrodes, before it was metalized with strips and prepared as a tracker.

\section{Irradiation Studies}

Radiation hardness is required for particle detectors in e.g. future particle physics experiments at the LHC/CERN or Tevatron/FNAL. In particular solid state tracking detectors have to resist high particle fluences keeping the signal-to-noise ratio as high as possible after irradiation. Solid state devices are damaged under particle irradiation. Damage in solid state detectors causes on one hand an increase in leakage current and therefore an increase in noise; on the other hand a reduction in the amount of collected charge, which leads to a smaller signal. The signalto-noise ratio in damaged detectors decreases. The potential advantage of diamond is the much larger dose that is apparently necessary to cause the same damage. 


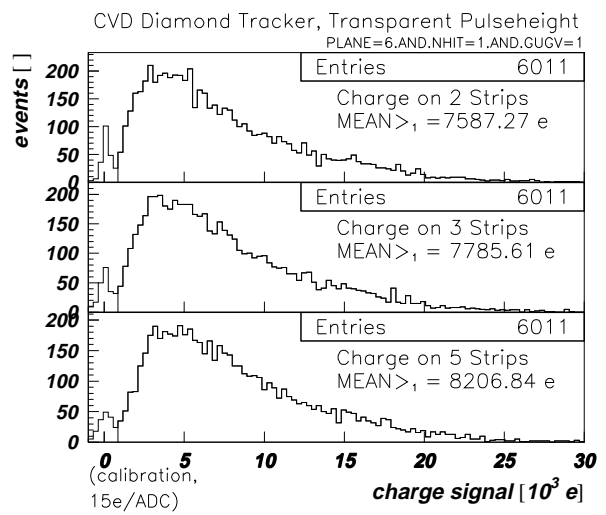

Figure 10: Pulse height distribution of the signal charge on strips next to the predicted track.

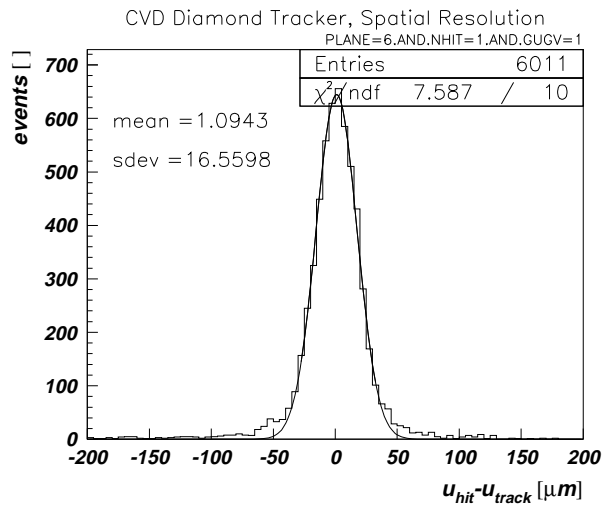

Figure 11: Distribution of the difference between the measured hit in the diamond detector and the predicted track intersection.

\subsection{Neutron Irradiation}

CVD diamond samples and silicon diodes were irradiated with neutrons in four exposures during the last two years using the ISIS facility at the Rutherford Appleton Laboratory, England. Protons from an accelerator interact in a graphite block and generate neutrons ranging from $10 \mathrm{keV}$ to several $10 \mathrm{MeV}$ peaking at $1 \mathrm{MeV}$ [10]. The mean $n$-flux above $10 \mathrm{keV}$ was about $(1.7 \pm 0.6) \times 10^{8} \mathrm{n} / \mathrm{cm}^{2} / \mathrm{s}$. The samples were kept at room temperature during irradiation.

The samples were biased at $100 \mathrm{~V}$ and we could measure their leakage current during irradiation [Fig. 12]. In diamond we observe a neutron or $\gamma$-background induced current $\approx 300 \mathrm{pA}$ which is correlated to the neutron flux. The neutron flux decreases as soon as the accelerator stops delivering protons to the spallation target which results in a prompt decreasing current in the diamond to a few pico-Ampere. On samples which were irradiated for the first time the induced current decreases exponentially and saturates on a level of several $100 \mathrm{pA}$. The leakage current in silicon diodes on the other side increases continuously from a few micro-Ampere to several $10 \mu \mathrm{A}$. During spill breaks when no neutrons are present one observes a slightly decreasing current in silicon (annealing). The charge signal distribution on a diamond samples before and after $8 \times 10^{14} \mathrm{n} / \mathrm{cm}^{2}$ is shown in Fig. 13 . The most probable value of the signal charge

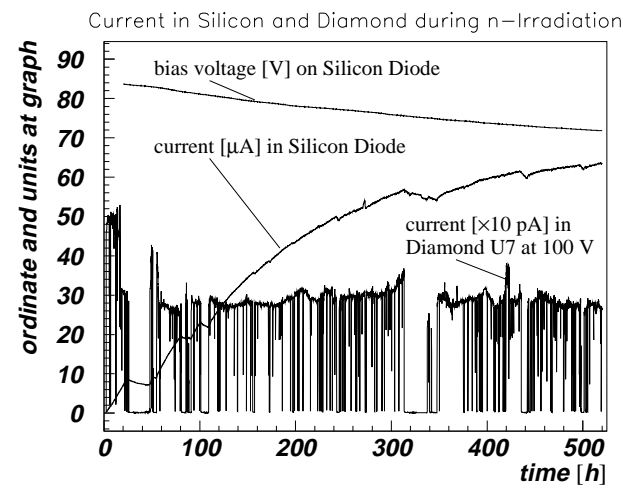

Figure 12: Induced current in diamond and in a silicon diode during neutron irradiation. The current in diamond shows a prompt response to the presence of neutrons. The induced current goes back to a few pico-Ampere during spill breaks when no neutrons are present. The current in Silicon increase continuously and anneals during spill breaks. does not change. The mean of the distribution after irradiation may have changed slightly but is within the measurement error compared to before irradiation. The charge collection distance normalized to the pumped value before irradiation is shown in Fig. 14. 


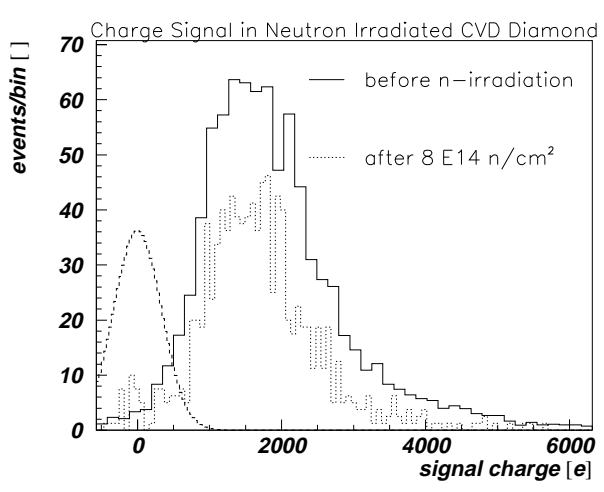

Figure 13: Charge signal distribution on a diamond detector sample before and after two $n$ irradiations. The sample acquired a $n$-fluence of $\approx 8 \times 10^{14} \mathrm{n} / \mathrm{cm}^{2}$.

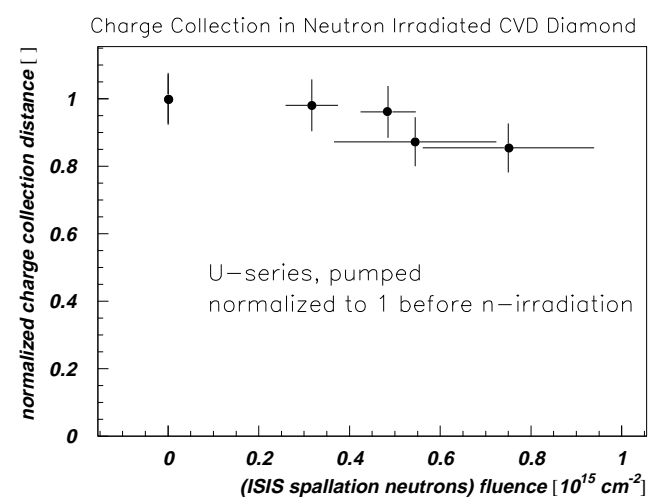

Figure 14: Charge collection distance in CVD diamond detector samples as a function of neutron fluence up to $8 \times 10^{14} \mathrm{n} / \mathrm{cm}^{2}$. The neutrons come from the ISIS spallation source and have a kinetic energy spectrum peaking at $1 \mathrm{MeV}$.

\subsection{Proton Irradiation}

CVD diamond samples were irradiated in 1995 with $500 \mathrm{MeV} / \mathrm{c}$ protons up to $8 \times 10^{13} \mathrm{p} / \mathrm{cm}^{2}$ at TRIUMF, Canada [11]. In 1997 other CVD diamond samples and one diamond strip detector were irradiated with $24 \mathrm{GeV} / c$ protons at the Proton Synchrotron (PS) at CERN.

The proton flux on samples went up to $1.1 \times 10^{10} \mathrm{p} / \mathrm{cm}^{2} / \mathrm{s}$ which allowed to irradiate up to a fluence of $5 \times 10^{15} \mathrm{p} / \mathrm{cm}^{2}$ in a reasonable time. The charge collection was measured at different proton fluences. As in the neutron irradiation we observe a particle induced current which correlates exactly with proton flux. Compared to the neutron irradiation the induced current has several microAmpere because protons interact electromagnetically with electrons in diamond. The charge collection distance at $1 \times 10^{15} \mathrm{p} / \mathrm{cm}^{2}$ shows the same value as before irradiation [Fig. 15]. The charge collection distance decreases linearly above $2 \times 10^{15} \mathrm{p} / \mathrm{cm}^{2}$ with a slope of $-14 \% / 10^{15} \mathrm{p} / \mathrm{cm}^{2}$.

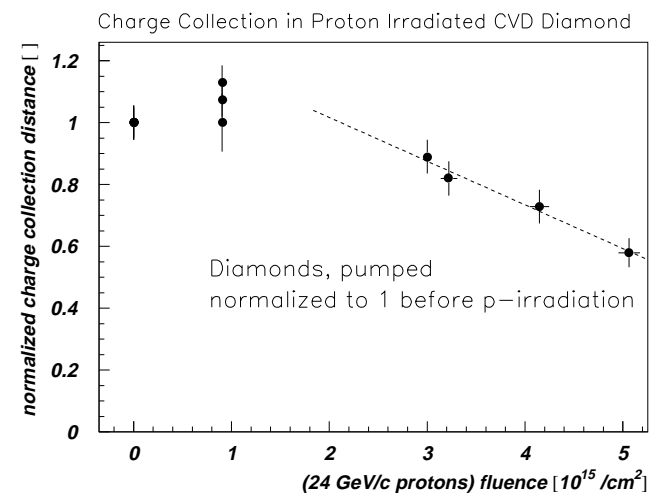

Figure 15: Charge collection distance in CVD diamond as a function of the proton fluence. The Protons had a momentum of $24 \mathrm{GeV} / c$.

\subsection{Pion Irradiation}

CVD diamond detector samples were irradiated with pions during the last three years at the PSI, Villigen, Switzerland [12]. The pions had a momentum of $300 \mathrm{MeV} / c$. The maximum available pion flux onto the samples was $\mathcal{O}\left(1.5 \times 10^{9}\right) \pi / \mathrm{cm}^{2} / \mathrm{s}$. During irradiation the samples were biased with a voltage of $100 \mathrm{~V}$ or above. The total pion fluence on samples irradiated until end of 1996 reached $1.7 \times 10^{15} \pi / \mathrm{cm}^{2}$. The normalized charge collection distance measured on CVD diamond samples after irradiation with pions is shown as a function of pion fluence in Fig. 16. The charge collection distance is normalized to the pumped state of the sample before irradiation. 
The samples remain in their pumped state after irradiation with pions and the sample which reaches the highest fluence of $1.7 \times 10^{15} \pi / \mathrm{cm}^{2}$ shows no decrease in collection distance. The dark current on these samples was measured before and after irradiation. A characteristic value is $0.5 \mathrm{pA} / \mathrm{mm}^{2}$ at $1 \mathrm{~V} / \mu \mathrm{m}$. The dark current is independent of the received pion fluence. These measurements indicate that the detector performance was unchanged after irradiation up to $1.7 \times 10^{15} \pi / \mathrm{cm}^{2}$.

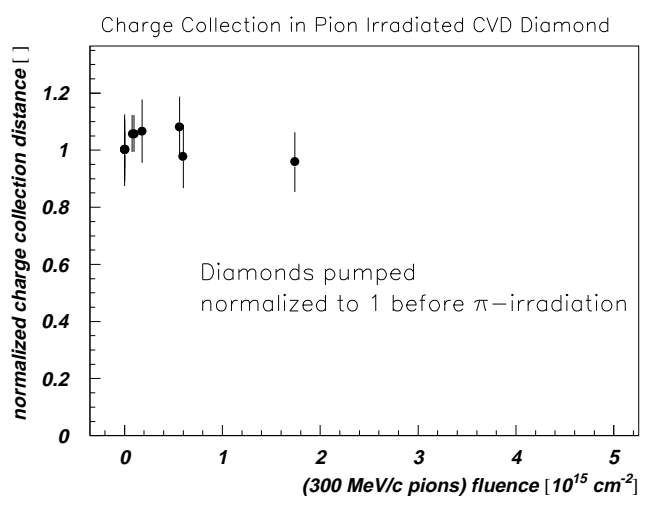

Figure 16: Charge collection distance in diamond as a function of the pion fluence. The pions had momentum of $300 \mathrm{MeV} / c$.

\section{Summary}

The charge collection distance on recent CVD diamonds now reaches $250 \mu \mathrm{m}$. The charge collection distance in CVD diamond can be improved by lapping the substrate side. The improvement is well predicted by the linear increase of charge collection distance along the direction of diamond growth. Charge collection distance increases (pumps) under illumination to charged particles. Traps are filled during pumping. The release of charge carriers from traps was observed by measuring either the current or luminescence light during heating. Both methods show either current or luminescence peaks at characteristic temperatures.

CVD diamonds were used to build micro-strip and pixel detectors for charged particle tracking. The mean collected charge from a recent tracker is $8200 e$ which corresponds to $230 \mu \mathrm{m}$ charge collection distance. The signal-to-noise is about 55-to-1. This tracker has a position resolution of $16.5 \mu \mathrm{m}$.

CVD diamond samples were exposed to neutrons, protons and pions in several irradiations over the last three years. After neutron irradiation up to $8.0 \times 10^{14} \mathrm{n} / \mathrm{cm}^{2}$ the mean of signal distribution may have changed slightly but is within the measurement error. Particle induced currents decrease during a first irradiation and saturate at a constant value up to the highest fluence. After pion irradiation no degradation in charge collection up to $1.7 \times 10^{15} \pi / \mathrm{cm}^{2}$ is observed. The leakage current after pion irradiation is the same as before irradiation. In a proton irradiation we find no decrease in charge collection distance up to $2 \times 10^{15} \mathrm{p} / \mathrm{cm}^{2}$ consistent with the pion irradiation result. The charge collection distance decreases above $2 \times 10^{15} \mathrm{p} / \mathrm{cm}^{2}$ with a slope of $-14 \% / 10^{15} \mathrm{p} / \mathrm{cm}^{2}$ up to $5 \times 10^{15} \mathrm{p} / \mathrm{cm}^{2}$.

We conclude that diamond is a radiation hard detector material which is able to withstand the conditions in high particle fluence regions of detectors at the LHC or Tevatron.

\section{Acknowledgements}

We would like to acknowledge the help from M. Edwards at RAL and the group from M. Glaser, F. Lemeilleur at CERN during $n$ and $p$-irradiation studies and the work on thermo-luminescence by K. Pajchel at CERN. 


\section{References}

[1] M.A. Plano et al., "Thickness Dependence of the Electrical Characteristics of Chemical Vapor Deposited Diamond Films", Appl. Phys. Lett. 64 (1994) 193.

[2] S. Zhao et al., "Electrical Properties in CVD Diamond Films", Mat. Res. Soc. Symp. 302 (1993) 257.

[3] The RD42 Collaboration, "R\&D Proposal, Development of Diamond Tracking Detectors for High Luminosity Experiments at the LHC", DRDC/P56, CERN/DRDC 94-21, (May 1994).

[4] S. Zhao, "Characterization of the Electrical Properties of Polycrystalline Diamond Films", Ph.D. Dissertation, Ohio State University (1994).

[5] W. Dulinski, "Electron Irradiation of CVD-Diamond", RD42 Collaboration Meeting Notes (Jun. 1995).

[6] D. Meier et al. (RD42-Collaboration), "It. Phys. Soc., Conference Proc. EuroDiamond 96", 52 (1996) 105.
[7] F. Borchelt et al. (RD42-Collaboration), "First Measurements with a Diamond Microstrip Detector", Nucl. Instr. and Meth. A354 (1995) 318.

[8] P. Apsell et al., "The VIKING readout chip", Nucl. Instr. and Meth. A315 (1992) 425 .

[9] The RD42 Collaboration, "Development of Diamond Tracking Detectors for High Luminosity Experiments at the LHC", LHCC Status Report/RD42, CERN LHCC 97-3 (Dec. 96).

[10] M. Edwards and D.R. Perry, "The ISIS Radiation Hardness Test Facility", RAL Report 90-065, p.21 (1990).

[11] S. Han et al. (RD42-Collaboration), "Proton Irradiation Studies of CVD Diamond Detectors", in preparation for submission to NIM (1996).

[12] C. Bauer et al. (RD42 Collaboration), "Pion Irradiation Studies of CVD Diamond Detectors", CERN-PPE/95-173, accepted for publication in NIM (1996). 